\title{
Analysis of the surface quality of polycaprolactam 3D prints enriched with carbon and glass fiber
}

\author{
Damian Dzienniak ${ }^{1 *}$, Jan Pawlik ${ }^{1}$ \\ ${ }^{1}$ Department of Manufacturing Systems, Faculty of Mechanical Engineering and Robotics, AGH \\ University of Science and Technology, Kraków, Poland
}

\begin{abstract}
Additive manufacturing has been gaining popularity and availability year by year, which has resulted in its dynamic development. The most common 3D printing method as of today, FDM (Fused Deposition Modeling), owing to its peculiarity, does not always guarantee producing objects with low surface roughness. The authors of the present article have taken on the analysis of the impact of FDM printing on the roughness of the filament thus processed. They also investigate the relationship between the roughness of the unprocessed filament (made of polycaprolactam, that is, polyamide 6 or PA6) with admixtures of other materials (carbon fiber, glass fiber) and the surface quality of the manufactured object. The main subject of the analysis is the side surfaces of $3 \mathrm{D}$ prints, as it is their quality that is usually directly dependent on many factors connected with the process of the laying of the consecutive layers. The authors check step by step whether there exists a pronounced relationship between the roughness of the original filament material and the roughness of the obtained surface.
\end{abstract}

\section{Introduction}

Additive manufacturing, commonly known as $3 \mathrm{D}$ printing, is an increasingly popular manufacturing process, during which layers of material are usually laid on top of one another [1]. The most common and widely available type of 3D printing is FDM [2], which stands for fused deposition modeling.

The ever-increasing availability of three-dimensional printing has caused it to find its application particularly in unit and small series production. Nevertheless, one of the problems that $3 \mathrm{D}$ printing still faces is the relatively poor accuracy of the obtained surface, which canin some demanding or specialized applications - be quite an obstacle. To a smaller or larger extent, a vast number of factors influence the quality of the obtained surface. Even the method or the printing parameters have a great impact on the quality of the final product, and the functional effectiveness of the product can significantly depend on the surface roughness level in the material [3]. The filament material itself can already be of great significance when it comes to the product quality, not only in terms of its surface. That is why research in this

\footnotetext{
* Corresponding author: ddamian@agh.edu.pl
} 
direction and attempts at combining materials are so important and interesting at the same time.

It may not always be desirable for a surface to have a low roughness-whether it is beneficial or not depends entirely on the final application of the product. For example, in the biomedical field, custom 3D-printed parts must often display a relatively high surface quality [4]. In the case of prototyping and printing larger parts, however, having rough surfaces is not always a problem. Moreover, for parts that have to fit tightly together, a rough surface may actually be advantageous.

With that being said, it is worth assessing the surface roughness of newly obtained materials for what it is before applying any additional processing. Then, depending on the intended use, a decision can be made whether the given 3D-printed object has a satisfactory surface quality.

Carbon fibers are commonly used as polymer additives, as they increase tensile strength while lowering mass and reducing thermal expansion [5]. On the other hand, it has been reported that admixtures of glass fibers can significantly increase the abrasion resistance of a material [6]. This provides another reason to investigate the surface quality of the novel materials.

\section{Materials and Methods}

\subsection{Samples}

For the purposes of this research, samples of filaments as well as 3D prints had been supplied by the ATMAT 3D printing company from Kraków, Poland. For surface quality tests, the following materials had been selected:

- pure PA6 (PA6 Natural),

- PA6 with a 20\% admixture of carbon fiber (PA6 CF20),

- PA6 with a 25\% admixture of glass fiber (PA6 GF25).

Microscope photos of the surface of each of the filaments are presented in Figure 1. The visual differences that cab be observed there were also to be reflected in the measurement results.

Table 1. 3D print parameters.

\begin{tabular}{|c|c|}
\hline Parameter & Value \\
\hline Layer Thickness & $0.15 \mathrm{~mm}$ \\
\hline Bottom Layer Thickness & $1 \mathrm{~mm}$ \\
\hline Wall Thickness & $1.6 \mathrm{~mm}$ \\
\hline Wall Line Count & 2 \\
\hline Infill & $30 \%$ \\
\hline Average Nozzle Temperature & $260^{\circ} \mathrm{C}$ \\
\hline Bed Temperature & $100^{\circ} \mathrm{C}$ \\
\hline
\end{tabular}


As for the printing parameters, in each of the instances, the layer thickness was set to $0.15 \mathrm{~mm}$. The infill was set to $30 \%$ and used the grid pattern. Table 1 lists also the rest of the important parameters.



Fig. 1. Microscope photos of the surfaces of the three different types of filaments (from left to right: PA6 Natural, PA6 20\% CF, PA6 25\% GF).

A photo of the samples is presented in Figure 2, whereas Figure 3 shows the side surface of each in greater detail. The nominal dimensions of each cube were $30 \mathrm{~mm} \times 30 \mathrm{~mm} \times 30$ $\mathrm{mm}$, although they may have differed slightly from the actual dimensions.
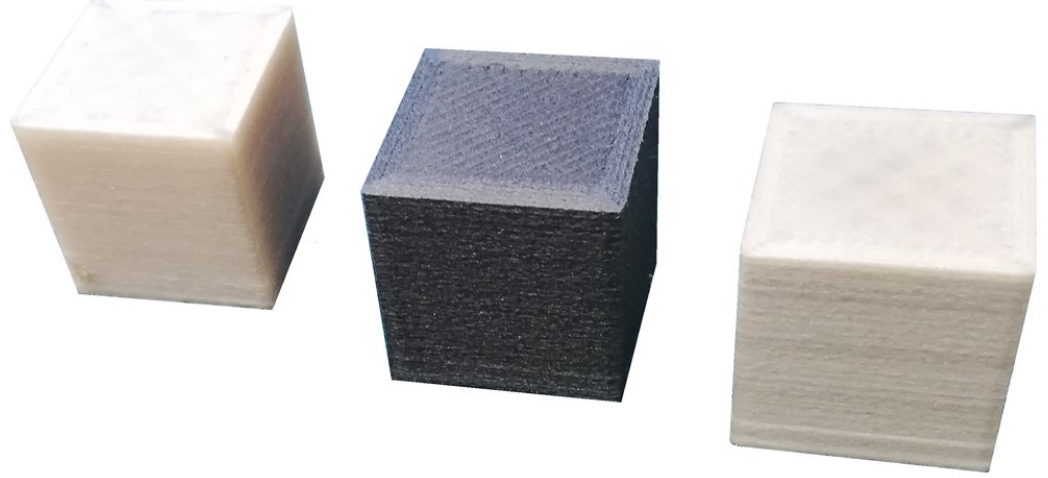

Fig. 2. The 3D-printed samples used for surface roughness assessment (from left to right: PA6 Natural, PA6 20\% CF, PA6 25\% GF).

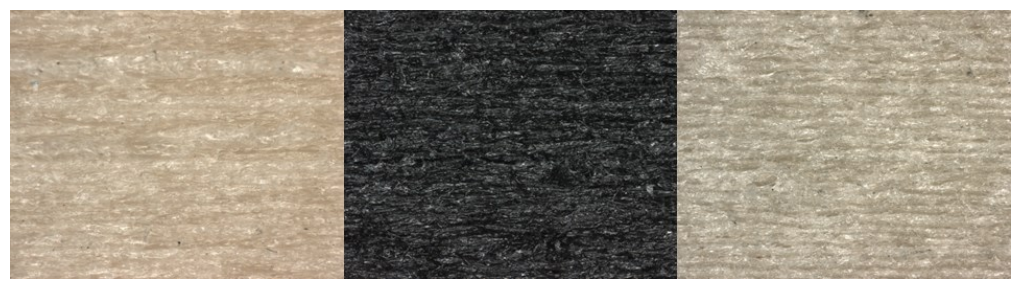

Fig. 3. Microscope photos of the surfaces of the 3D-printed samples (from left to right: PA6 Natural, PA6 20\% CF, PA6 25\% GF).

\subsection{Surface Roughness}

The $R_{a}$ parameter expresses the arithmetic mean deviation of the roughness profile relative to its center line. In practice, it is usually computed for a finite set of values, which can be approximated by a sum, as in Eq. 1

$$
R_{a}=\frac{1}{l} \int_{0}^{l}|z(x)| d x \approx \frac{1}{n} \sum_{i=1}^{n}\left|z_{i}\right|
$$


where $l$ is the evaluation length, and $z(x)$ is the distance from the mean line to the measurement point on the surface profile. The discrete version replaces the evaluation length with the number of samples, $n$.

The $R_{q}$ parameter, known as the root mean square (RMS) deviation of the roughness profile, is expressed by Eq. 2. The rest of the symbols are the same as in the previous equation.

$$
R_{q}=\sqrt{\frac{1}{l} \int_{0}^{l} z^{2}(x) d x} \approx \sqrt{\frac{1}{n} \sum_{i=1}^{n}\left|z_{i}^{2}\right|}
$$

The 10-point mean roughness height, $R_{z}$, is the sum of the mean value of the five highest peaks and the mean value of the five deepest valleys within the evaluation length 1 . More specifically, it is computed according to Eq. 3

$$
R_{z}=\frac{1}{5}\left(\sum_{i=1}^{5}\left|z_{p i}\right|+\sum_{i=1}^{5}\left|z_{v i}\right|\right)
$$

where $z_{p i}$ corresponds to the five highest profile peaks, and $z_{v i}$ corresponds to the five deepest profile valleys over the evaluation length.

These parameters and corresponding formulae are included in the international standards such as ISO 4287-1997 and ASME B46.1-2002 [3], even though currently, the $R_{z}$ parameter is no longer a part of the new ISO standard.

\section{Measurements}

All contact surface roughness measurements were performed using the Formtracer SVC4500 surface and contour measuring instrument equipped with the SV-C3000 roughness detector, shown in Figure 4, both of which were manufactured by Mitutoyo. The measurement length in each case was set to $4.8 \mathrm{~mm}$, the evaluation length to $4 \mathrm{~mm}$, and parameters describing surface roughness such as $R_{a}, R_{q}$, and $R_{z}$ were computed by the software provided with the measuring system. The distance between the consecutive points was $0.0005 \mathrm{~mm}$, and the sampling length was set to $0.8 \mathrm{~mm}$. The system comes with software called Formtracepak, which facilitates the acquisition and processing of measurement results. For the sake of readability, the selected measurement parameters have also been listed in Table 2.

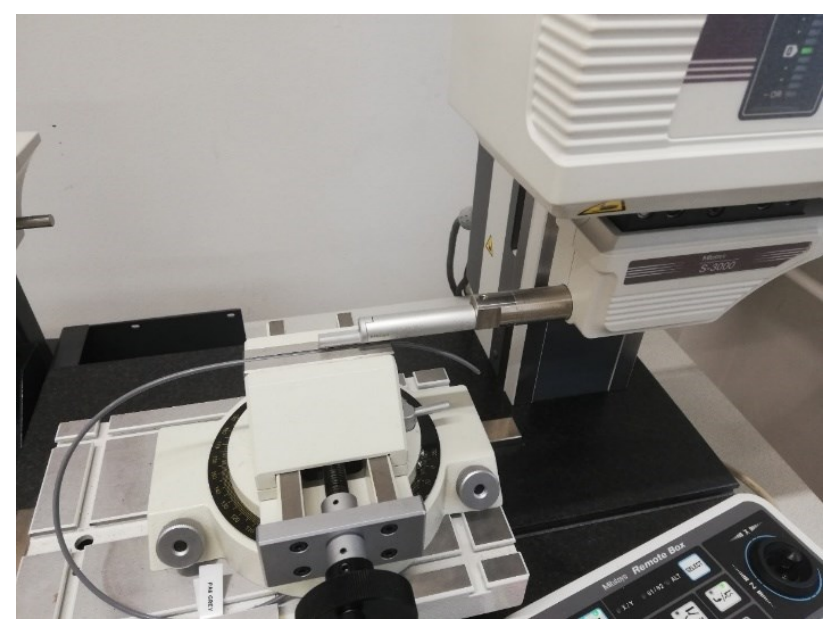

Fig. 4. The stand for contact measurements of surface roughness. 
Table 2. Roughness measurement parameters.

\begin{tabular}{|c|c|}
\hline Parameter & Value $[\mathbf{m m}]$ \\
\hline Measurement Length & 4.8 \\
\hline Evaluation Length & 4 \\
\hline Sample Length & 0.8 \\
\hline Step Size & 0.0005 \\
\hline
\end{tabular}

The roughness of the filaments provided was measured 12 times for each of them, in 12 different locations, to avoid obtaining unreliable results (for example, due to potential local mechanical damages, such as scratches or buildups of dirt). The same approach was applied in the case of measuring the surface roughness of the 3D-printed cubes. Likewise, the roughness was measured in three different locations on each of the four sides of every cube. Six of the measurements for each cube were performed with the stylus moving in one direction, and another six measurements - in the other direction. Again, this was supposed to ensure that the results were not just a product of mere chance.

Furthermore, two approaches were employed to measure the roughness of the sides. The first one consisted in - as would normally be done - measuring with the stylus of the detector moving along the path that would yield the highest possible roughness, and that, naturally, would be perpendicular to the layers. However, this method is likely to be affected by such factors as the desired layer thickness, since with an increasing number of layers, the overall surface roughness may decrease. In general, roughness values may vary depending on the measuring direction [7]. The other approach was to measure the roughness along the 3Dprinted layers. This helps us assess the roughness of the processed filament, minimizing the impact of how the layers are laid or interwoven, and even if it is not $100 \%$ accurate, it definitely gives us a much better idea about the roughness of the processed material itself.

\section{Results}

The results of the roughness measurements of the filaments are presented numerically in Tables 3-6, as well as graphically in Figures 5-8. As we can see, the natural PA6 filament has a much lower surface roughness than the other two materials. Table 3 and Figure 5 contain the results for the unprocessed filaments. Table 4 and Figure 6 show the averaged-out results for the roughness measurements taken across the layers of the sample cubes. Next, Table 5 and Figure 7 contain the results obtained while measuring the roughness along the layers. Finally, Table 6 and Figure 8 present the ratio between the average surface roughness of the 3D-printed samples and the average surface roughness of the corresponding filaments.

Table 3. Roughness parameters obtained for the unprocessed filaments.

\begin{tabular}{|c|c|c|c|}
\hline Material & $\mathbf{R a}[\boldsymbol{\mu m}]$ & $\mathbf{R q}[\boldsymbol{\mu m}]$ & $\mathbf{R z}[\boldsymbol{\mu m}]$ \\
\hline PA6 Natural & 0.325 & 0.415 & 2.340 \\
\hline PA6 CF20 & 5.571 & 6.794 & 27.139 \\
\hline PA6 GF25 & 5.449 & 6.553 & 24.369 \\
\hline
\end{tabular}




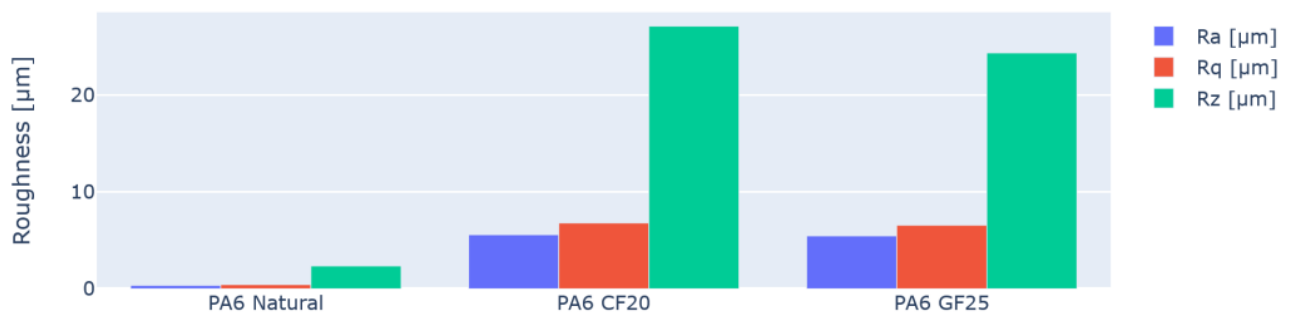

Fig. 5. A graphical representation of the average surface roughness measured along the respective filament types.

Table 4. Roughness parameters obtained for the 3D-printed cubes measured across the layers.

\begin{tabular}{|c|c|c|c|}
\hline Material & $\mathbf{R a}[\boldsymbol{\mu m}]$ & $\mathbf{R q}[\boldsymbol{\mu m}]$ & $\mathbf{R z}[\boldsymbol{\mu m}]$ \\
\hline PA6 Natural & 21.279 & 25.468 & 100.645 \\
\hline PA6 CF20 & 22.698 & 27.781 & 118.502 \\
\hline PA6 GF25 & 19.360 & 24.008 & 103.703 \\
\hline
\end{tabular}



Fig. 6. A graphical representation of the average surface roughness measured across the layers of the cubes.

Table 5. Roughness parameters obtained for the 3D-printed cubes measured along the layers.

\begin{tabular}{|c|c|c|c|}
\hline Material & $\mathbf{R a}[\boldsymbol{\mu m}]$ & $\mathbf{R q}[\boldsymbol{\mu m}]$ & $\mathbf{R z}[\boldsymbol{\mu m}]$ \\
\hline PA6 Natural & 6.335 & 8.054 & 34.394 \\
\hline PA6 CF20 & 12.837 & 16.398 & 72.513 \\
\hline PA6 GF25 & 11.606 & 15.063 & 67.274 \\
\hline
\end{tabular}






Fig. 7. A graphical representation of the average surface roughness measured along the layers of the cubes.

Table 6. The ratio of the roughness values measured along the layers and the roughness of the unprocessed filaments.

\begin{tabular}{|c|c|c|c|}
\hline Material & $\mathbf{R a}$ & $\mathbf{R q}$ & $\mathbf{R z}$ \\
\hline PA6 Natural & 19.468 & 19.398 & 14.696 \\
\hline PA6 CF20 & 2.304 & 2.414 & 2.672 \\
\hline PA6 GF25 & 2.130 & 2.299 & 2.761 \\
\hline
\end{tabular}

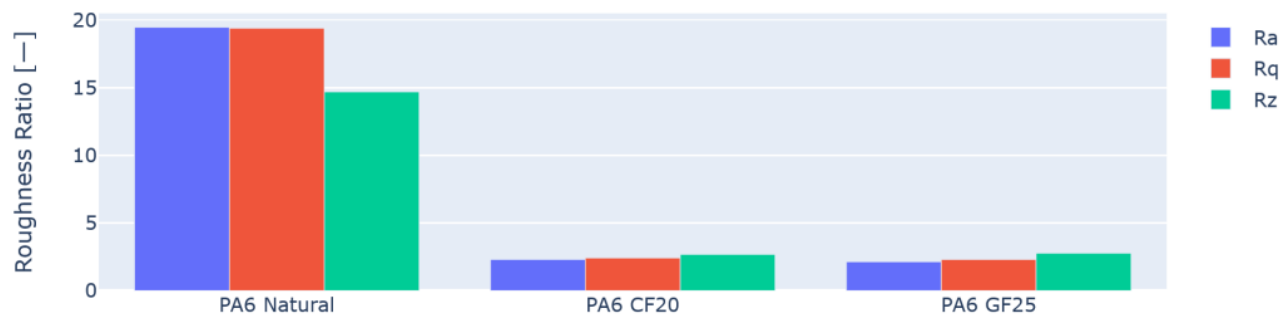

Fig. 8. A graphical representation of the ratio between the average surface roughness of the 3Dprinted samples and the average surface roughness of the corresponding filaments.

\section{Discussion and Conclusions}

The PA6-based filaments with the admixtures were characterized by roughness even up to 10 times higher than that of PA6 with no admixture. The roughness of the side surfaces of the prints is still lower for pure PA6 in comparison with the prints containing carbon fiber and glass fiber, albeit more or less twice as low. The percent increase in the roughness of the $3 \mathrm{D}$ print relative to the roughness of the filament was much lower in the case of PA6 CF20 and PA6 GF25 than that in the case of the natural filament made of polycaprolactam. The result of the contact roughness measurement may be influenced by many factors, even though optical measurements in that case would not necessarily be easy.

It can also be concluded that even though the roughness of the materials enhanced with carbon and glass fibers is generally higher than that of the natural material, it does not necessarily mean that there will be no application for them. Furthermore, there may be aspects in which those materials will have their advantages, which can constitute the object of further studies. 


\section{References}

1. H. Nieciąg, R. Kudelski, P. Dudek, J. Cieślik, Acta Mech. et Autom. 14, 1 (2020)

2. V. Mazzanti, L. Malagutti, F. Mollica, Polymers 11, 7 (2019)

3. B.N. Turner, S.A. Gold, Rapid Prototyp. J. 21, 3 (2015)

4. J. H. Kim, M. Y. Kim, J. C. Knowles, S. Choi, H. Kang, S. H. Park, S. M. Park, H. W. Kim, J. T. Park, J. H. Lee, H. H. Lee, Dent. Mater. 36, 7 (2020)

5. S. F. A. Acquah, B. E. Leonhardt, M. S. Nowotarski, J. M. Magi, K. A. Chambliss, T. E. S. Venzel, S. D. Delekar, L. A. Al-Hariri, Carbon Nanotubes: Current Progress of Their Polymer Composites, 8 (IntechOpen, 2016)

6. S. Kumar, K. Panneerselvam, Proc. Technol. 25, pp. 1129-1136 (2016)

7. M. S. Alsoufi, A. E. Elsayed, Am. J. Mech. Eng., 5, 5 (2017) 